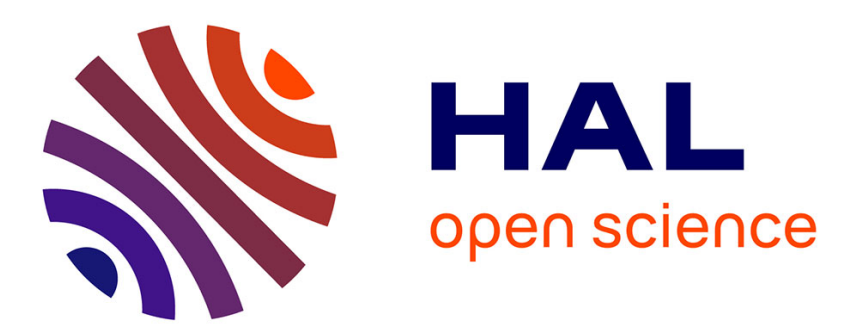

\title{
Forecasting VaR and Expected Shortfall using Dynamical Systems: A Risk Management Strategy
}

Cyril Caillault, Dominique Guegan

\section{To cite this version:}

Cyril Caillault, Dominique Guegan. Forecasting VaR and Expected Shortfall using Dynamical Systems: A Risk Management Strategy. Frontiers in finance and economics, 2009, 6 (1), pp.26-50. halshs-00375765

\section{HAL Id: halshs-00375765 https://shs.hal.science/halshs-00375765}

Submitted on 16 Apr 2009

HAL is a multi-disciplinary open access archive for the deposit and dissemination of scientific research documents, whether they are published or not. The documents may come from teaching and research institutions in France or abroad, or from public or private research centers.
L'archive ouverte pluridisciplinaire HAL, est destinée au dépôt et à la diffusion de documents scientifiques de niveau recherche, publiés ou non, émanant des établissements d'enseignement et de recherche français ou étrangers, des laboratoires publics ou privés. 


\title{
Forecasting VaR and Expected Shortfall using Dynamical Systems: A Risk Management Strategy
}

\author{
Cyril Caillault ${ }^{*}$
}

Dominique Guégan ${ }^{2}$

\begin{abstract}
Using non-parametric and parametric models, we show that the bivariate distribution of an Asian portfolio is not stable along all the period under study. We suggest several dynamic models to compute two market risk measures, the Value at Risk and the Expected Shortfall: the RiskMetrics methodology, the Multivariate GARCH models, the Multivariate Markov-Switching models, the empirical histogram and the dynamic copulas. We discuss the choice of the best method with respect to the policy management of bank supervisors. The copula approach seems to be a good compromise between all these models. It permits taking financial crises into account and obtaining a low capital requirement during the most important crises.
\end{abstract}

Keywords: Value at Risk, Expected Shortfall, Copulas, Risk management, GARCH models, Markov switching models.

JEL classification: C15, C32, C52, G28.

\footnotetext{
Chief Investor Officer, Quantitative Strategies, Fixed Income and Currencies, Fortis Investments, 82, Bishopsgate London EC2N4BN United Kingdom. Tel. +44 207063 72 30. e-mail: cyril.caillault@fortisinvestments.com

${ }^{2}$ PSE - CES-MSE - Université Paris 1 - Panthéon - Sorbonne, 106 - 112 boulevard de l'hôpital, 75647 Paris Cedex 13 Tel : +33 (0)1 44078298 - e-mail: dguegan@univ-paris1.fr.
} 
Cyril Caillault, Dominique Guégan - Forecasting VaR and Expected Shortfall using Dynamical Systems: A Risk Management Strategy - Frontiers in Finance and Economics - Vol. 6 No.1 - April 2009,

\section{1 - Introduction}

The main objective of the 1988 Basel Accord is to develop a riskbased capital framework that strengthens and stabilises the banking system. In 1996, this accord was revised in order to take into account the importance of market risk. Capital requirements are a common aspect of the regulations of financial institutions. They were implemented with a list of standardized rules that appear simple and robust but have the drawback to not be sensitive enough to the risk profile of the institutions. By now, capital requirements are increasingly based on risk-sensitive measures, which are directly linked to VaR (Value-at-Risk) for market risk. VaR is now a common language to describe market risk for different type of assets such as equities, rates or currencies. The VaR measure, which may be expressed in cash amount, can be translated directly into a minimum capital requirement, BCBS (1996). This text has leaded the financial institutions to develop their own internal VaR model. Nevertheless, even if all the pillars are in place to quantitatively calculate VaR, there is no consensus on the VaR model specifications. This suggests that financial institutions are free to use the model for which they are comfortable the most but not necessarily the model that describes the best the data. In this paper, we demonstrate that the choice of the model can provide a very different picture of risk. On the other hand, VaR is based on some unrealistic assumptions. Specifically, it does not verify the sub-additivity property of a coherent risk measure, therefore the ES (Expected Shortfall) measure is superior and is preferable; see for instance Artzner at al. (1997) and Yamai and Yoshida (2002) for a description of the VaR weaknesses.

In this paper, we are interested in comparing the effect of these two risk measures - VaR and ES - to measure the risk associated to a twodimensional portfolio. This portfolio will be composed of the returns of three Asian composite indexes - the daily closing level of the Thai SET index, the Malaysian KLCI index and the Indonesian JCI index. A preliminary statistical study of these indexes permits detection of specific features inside the data sets including heteroscedasticity and switches. The analysis of the evolution of the marginal distribution function of each return shows the existence of non-stationarity that is now a well known problem inside this kind of data set, Mikosch and Starica (2004) and Guégan (2007b). In order to take these kinds of patterns into account, we calculate the two risk measures using parametric models like the RiskMetrics model, RiskMetrics (1996), the GARCH models, Bollerslev (1986) and the Markov switching processes, Hamilton (1988). We also consider a non parametric approach using the copula method which 
allows us to calculate the bivariate distribution function of the portfolio in a dynamic way.

In the literature there already exist many studies on the computation of the VaR of a portfolio using the previous parametric models. We can cite, for instance, Guermat and Harris (2001), Huang and Lin (2004) and Rodriguez (2004). The copula approach has also been extensively used to compute the VaR, Malevergne and Sornette (2003), Rodriguez (2004), Caillault and Guégan (2005). At the same time several authors have pointed out the problem of non stationarity of the bivariate distribution function of a two-dimension portfolio and investigated this new domain of research. We specifically think of the works of Patton (2001), Dias and Embrechts (2003), Fermanian (2005), Patton, Granger and Terasvirta (2006), Jondeau and Rockinger (2006) and Guégan and Zhang (2009).

In this paper, our method appears slightly different from all these works, associating both parametric and non-parametric methods in a dynamic way. It gives a more complete description of the different approaches for both of the risk measures (VaR and ES). This is new in the literature and introduces a greater flexibility for the policy management of the bank supervisors.

This paper is organized as follows. In Section two, we first recall the definitions of the VaR and ES measures. Then, we present different methods to compute these measures, in a dynamic way, for a two-dimensional portfolio: both parametric and non-parametric models. In Section three, we introduce the data sets. We estimate the distribution function and the models associated to each return. Then, we analyse the two-dimensional portfolio's distribution using copulas on different samples. Finally, for each portfolio, we specify the estimated GARCH and Markov switching models. In Section four, we compute the VaR and ES measures on different windows using the previous models adjusted to the three two-dimensional portfolios. The results are also compared with those obtained under a stationary assumption. In Section five we discuss the choice of the best method with respect to the policy management of bank supervisors in order to make risk management decisions. 


\section{2 - VaR and Expected Shortfall measures}

In this Section we present different models to compute the $\mathrm{VaR}$ and the ES risk-measures. We denote by $P$ the portfolio of the log-returns $\left(R_{t+1}\right)_{t+1}$ defined as

$$
R_{t+1}=a^{\prime} r_{t+1}
$$

where $r_{t+1}=\left(r_{i, t+1}, r_{j, t+1}\right)^{\prime}, i, j=1,2,3,^{3}$ is a $2 \times 1$ vector of risk factors, and $a$ is a $2 \times 1$ vector of portfolio weights such that $\sum_{i=1}^{2} a_{i}=1$.

The VaR measure associated to the portfolio $P$, at date $t+1$, for a given $\alpha$, is denoted $\operatorname{VaR}_{\alpha}^{t+1}$ and satisfies:

$$
\operatorname{Pr}\left[R_{t+1} \leq \operatorname{VaR}_{\alpha}^{t+1}\right]=\alpha
$$

Thus, $\operatorname{VaR}_{\alpha}^{t+1}$ is the $\alpha$-quantile of the distribution function of the returns $R_{t+1}$ which depends on the $r_{t+1}$ multivariate distribution function. Thus, the VaR is simply the maximum loss that is encountered over a specified period with a level of confidence 1- $\alpha$. Losses lower than $\operatorname{VaR}_{\alpha}$ occur with probability $\alpha$, here at time $\mathrm{t}+1$.

The Expected Shortfall measure associated to $R_{t+1}$, at time $\mathrm{t}+1$, denoted $E S_{\alpha}^{t+1}$, is:

$$
E S_{\alpha}^{t+1}=E\left(R_{t+1} \mid R_{t+1} \leq \operatorname{VaR}_{\alpha}^{t+1}\right)
$$

In (3), the Expectation is taken with respect to the distribution function of the returns $R_{t+1}$. This coherent measure represents the expectation

\footnotetext{
${ }^{3} x^{\prime}$ stands for transpose of vector $x$.
} 
of loss knowing that a threshold is exceeded, here $V_{a} R_{\alpha}$. It is a minorant of $V_{a} R_{\alpha}$ introduced in (2). We note that $E S_{\alpha}$ is also called the Conditional Value at Risk (CVaR) in some papers, for example Rochafellar and Uryasev (2002).

In the following, we compute these two quantities (2) and (3) using five approaches: the RiskMetrics method, the multivariate GARCH model with Gaussian and Student- $t$ innovations, the multivariate Markov-Switching models, the Empirical Histogram method and the copulas' method. The first three methods are based on the specification of the conditional joint distribution of the process $\left(r_{t+1}\right)_{t+1}$ whereas the fourth one uses the empirical distribution of the process $\left(R_{t+1}\right)_{t+1}$ and the last one uses the unconditional distribution of the process $\left(r_{t+1}\right)_{t+1}$ obtained via the copula method.

Throughout this paper we only compute the maximum potential loss for a portfolio $P$ over a given time horizon $\tau=1$ because we work with daily log-returns of Asian indexes. Nevertheless, several authors have discussed the choice and the effects of large $\tau$, see for instance Christoffersen et al. (1998) and Brummelhuis and Guégan (2005).

\subsection{VaR and ES measures computed using parametric models}

$\operatorname{VaR}_{\alpha}^{t+1,(\text { method })}$ is the $\mathrm{VaR}$ calculated with one of the following methods whose expressions are given by the formulae (6), (8), (9) and (12) below. $E S_{\alpha}^{t+1}$ is obtained using the following relationship:

$$
E S_{\alpha}^{t+1}=\left(\sum_{i=1}^{t+1} 1_{\left\{R_{i} \leq V a R_{\alpha}^{t+1,(\text { method })}\right\}}\right)^{-1} \sum_{i=1}^{t+1} R_{i} 1_{\left\{R_{i} \leq V a R_{\alpha}^{t+1,(\text { method })}\right\}} .
$$

$1_{\{.\}}$denotes the indicator function and it is equal to one if its argument is true and zero otherwise. 


\subsubsection{The RiskMetrics approach}

This method, also called the EWMA (exponentially weighted moving average) approach, was introduced by RiskMetrics (1996). Here, we assume that the returns $\left(r_{t+1}\right)_{t+1}$ are characterized by a joint conditional Gaussian distribution, mean 0 and time varying variance-covariance matrix $\Sigma_{t+1}=\left(\sigma_{i j, t+1}^{2}\right)_{i, i=1,2}$. The components of the matrix $\Sigma_{t+1}$ are computed via:

$$
\sigma_{i j, t+1}^{2}=\lambda \sigma_{i j, t}^{2}+(1-\lambda) r_{i, t} r_{j, t}
$$

where $\lambda$ is the decay factor, taken to be 0.94 by RiskMetrics. Then, for a given $\alpha, \operatorname{VaR}_{\alpha}^{t+1}$ is equal to:

$$
\operatorname{VaR}_{\alpha}^{t+1}=q_{\alpha} \sigma_{t+1}
$$

with $\sigma_{t+1}=\sqrt{a^{\prime} \Sigma_{t+1} a}$ and $q_{\alpha}$ the $\alpha$-quantile of the standard Gaussian distribution.

\subsubsection{The GARCH approach}

In order to take into account the volatility of the data set, we adjust a multivariate GARCH model on the returns $\left(r_{t+1}\right)_{t+1}$. We denote this model DVEC, Bollerslev et al. (1988). It is defined by:

$$
\left\{\begin{array}{l}
r_{t+1}=c+\varepsilon_{t+1} \\
\varepsilon_{t+1}=V_{t+1}^{1 / 2} Z_{t+1} \\
V_{t+1}=A+\sum_{i=1}^{p} A_{i} \otimes\left(\varepsilon_{t+1-i} \varepsilon_{t+1-i}^{\prime}\right)+\sum_{j=1}^{q} B_{i} \otimes V_{t+1-i}
\end{array},\right.
$$


where $V_{t+1}$ is the conditional covariance matrix of the $2 \times 1$ vector $\left(\varepsilon_{t+1}\right)_{t+1}$. $A, A_{i}$ and $B_{j}$ are $2 \times 2$ symmetric matrices, $c$ is a $2 \times 1$ vector of constants such that $c=(C(1), C(2))^{\prime}$. The symbol $\otimes$ stands for the Hadamard product.

The conditional standard deviation of the returns $\left(R_{t+1}\right)_{t+1}$ is $v_{t+1}=\sqrt{a^{\prime} V_{t+1} a}$. Then:

if the strong white noise $Z_{t+1}$ in (7) is a multivariate Gaussian distribution, mean 0 and variance $1, \operatorname{VaR}_{\alpha}^{t+1}$ is equal to:

$$
\operatorname{VaR}_{t+1}^{\alpha}=q_{\alpha} v_{t+1}+a^{\prime} c
$$

- $\quad$ if the strong white noise $Z_{t+1}$ follows a multivariate Student- $t$ distribution with $v$ degrees of freedom, then:

$$
\operatorname{VaR}_{t+1}^{\alpha}=q_{v, \alpha} \sqrt{(v-2) / v} v_{t+1}+a^{\prime} c,
$$

where $q_{v, \alpha}$ is the $\alpha$-quantile of the multivariate Student- $t$ distribution.

\subsubsection{Markov-Switching approach}

In order to take volatility and jumps inside the returns into account, we use a multivariate Markov-Switching model, Francq and Zakoian (2001). Thus, the return $\left(r_{t+1}\right)_{t+1}$ depends on a state variable $s_{t+1}$ that is assumed to be an ergodic Markov chain with $l$ states. In the following $l=2$ or $l=3$. Then, the multivariate process associated to $\left(r_{t+1}\right)_{t+1}$ is defined by the following scheme on each state:

$$
r_{t+1}-\mu_{s_{t+1}}=\sum_{i=1}^{p} \gamma_{i}\left(r_{t+1-i}-\mu_{s+1-i}\right)+\varepsilon_{t+1},
$$


where $\mu_{s_{t+1}}$ is a $l \times 1$ vector of mean returns in state $s_{t+1}, \quad \gamma_{i}$ is an $l \times 1$ constant matrix and $\left(\varepsilon_{t+1}\right)_{t+1}$ is a Gaussian white noise with variancecovariance matrix $\Lambda_{s_{t+1}}$. The Markov chain $\left(s_{t+1}\right)_{t+1}$ is characterized by its transition probability matrix $P=\left(p_{i j}\right)_{i, j=1, \ldots, l}$ :

$$
p_{i j}=\operatorname{Pr}\left[s_{t+1}=i \mid s_{t}=j\right], i, j=1, \ldots, l .
$$

When the process $\left(r_{t+1}\right)_{t+1}$ follows the model (10), $\operatorname{VaR}_{\alpha}^{t+1}$ is obtained numerically solving:

$$
\begin{gathered}
\alpha=\sum_{s_{t+1}=1}^{l} \operatorname{Pr}\left[s_{t+1} \mid R_{1}, \ldots, R_{t}\right] \times \cdots \\
\int_{-\infty}^{\operatorname{VaR}_{\alpha}^{t+1}} N\left(x, a^{\prime} \mu_{t+1}, a \Lambda_{s_{t+1}} a \mid R_{1}, \ldots, R_{t}\right) d x
\end{gathered}
$$

Here, $\operatorname{Pr}\left[s_{t+1} \mid R_{1}, \ldots, R_{t}\right]$ represents the filtered probabilities and $N$ is the Gaussian distribution with mean $a^{\prime} \mu_{t+1}$ and standard deviation $a^{\prime} \Lambda_{s_{t+1}} a$.

\subsection{VaR and ES measures obtained from the Empirical Histogram}

The simplest way to compute the VaR measure is based on the empirical distribution of the portfolio $P$. Assume that, at the end of day $t$, we have $t$ observations for $P$. In this case, the VaR measure available for the next day $t+1$ is defined as the $\alpha$-quantile considered in the left end side of the empirical distribution of $P$. A numerical way to obtain this quantile is to arrange the $t$ observations in ascending order and to take the $\alpha$-th component of this vector. Then, $\operatorname{VaR}_{\alpha}^{t+1}$ is equal to:

$$
\operatorname{VaR}_{\alpha}^{t+1}=R_{(\alpha t), t},
$$

where $R_{(i), t}$ represents the $i$-th component of the order statistic. If $\alpha t$ is not an integer, then we applied an interpolation method between the two components $R_{e(\alpha t), t}$ and $R_{e(\alpha t+1), t}$. Here $e($.$) is the integer part function.$ 
We obtained $E S_{\alpha}^{t+1}$ by taking the average of the log-returns that are lower than $\operatorname{VaR}_{\alpha}^{t+1}$ :

$$
E S_{\alpha}^{t+1}=\frac{1}{\alpha t} \sum_{i=1}^{\alpha t} R_{(i), t}
$$

In the expression (14) all the observations have the same weights.

\subsection{VaR and ES measures computed using copulas}

In order to introduce the notion of copula, we denote by $F$ the unconditional cumulative joint distribution of the returns $\left(r_{t+1}\right)_{t+1}$. Sklar (1959) shows that a cumulative distribution function (cdf) of dimension 2 may be decomposed into 2 marginals and a copula. We denote by $F_{1}$ and $F_{2}$ the cdf of the returns $r_{1, t+1}$ and $r_{2, t+1}$ respectively. Then, if $F_{1}$ and $F_{2}$ are continuous, there exists an unique copula $C_{\theta}$ defined on $[0,1] \times[0,1]$, depending on a parameter $\theta$, such that:

$$
\forall r_{1, t+1}, r_{2,+1} \in \Re, F\left(r_{1, t+1}, r_{2,+1}\right)=C_{\theta}\left(F_{1}\left(r_{1, t+1}\right), F_{2}\left(r_{2, t+1}\right)\right) .
$$

Using the expression (15) we can derive the univariate cdf, denoted $G_{R_{t+1}}$, for the portfolio $P$. It satisfies:

$$
G_{R_{t+1}}(r)=\iint_{R_{t+1} \leq r} d C_{\theta}\left(F_{1}\left(a_{1} r_{1, t+1}\right), F_{1}\left(a_{2} r_{2, t+1}\right)\right) .
$$

$\operatorname{VaR}_{\alpha}^{t+1}$, which is the $\alpha$-quantile computed using the $G_{R_{t+1}}$ distribution associated to the portfolio $P$, is obtained numerically by solving the following equation:

$$
G_{R_{t+1}}\left(V_{a} R_{\alpha}^{t+1}\right)=\alpha .
$$

Then, the expected shortfall is given by: 


$$
\begin{aligned}
E S_{\alpha}^{t+1} & =E\left(R_{t+1} 1_{\left\{R_{t+1} \leq V a R_{\alpha}^{t+1}\right\}}\right) / E\left(1_{\left\{R_{t+1} \leq V a R_{\alpha}^{t+1}\right\}}\right) \\
& =\alpha^{-1} \iint_{\mathfrak{R}^{2}} R_{t+1} 1_{\left\{R_{t+1} \leq V a R_{\alpha}^{t+1}\right\}} d C_{\theta}\left(F_{1}\left(a_{1} r_{1, t+1}\right), F_{1}\left(a_{2} r_{2, t+1}\right)\right)
\end{aligned}
$$

Notice that the $\operatorname{VaR}_{\alpha}^{t+1}$ and $E S_{\alpha}^{t+1}$ measures depend on the margins of the returns and also on the copula that characterizes their joint distribution function. To compute the VaR and the ES measures, we need to solve the expressions (17) and (18). This is not always an easy task due to the nonconvex nature of the problems and in Section 4, Monte Carlo simulations are used to reconstruct the copulas and the distribution of the portfolio $P$.

\section{3 - Analysis and modelling of the data sets}

In this Section, we consider the returns of three Asian composite indexes which consist of the daily closing level of the Thai SET index $\left(r_{1, t}\right)_{t}$, the Malaysian KLCI index $\left(r_{2, t}\right)_{t}$ and the Indonesian JCI index $\left(r_{3, t}\right)_{t}$. These returns correspond to $r_{i, t+1}=\ln \left(W_{i, t+1}\right)-\ln \left(W_{i, t}\right)$, where $W_{i, t}$ is the level price (expressed in Japanese currency) at date $t$ for the three indexes $i=1,2,3$. The data sets start from July 2, 1987 and finish December 17, 2002. Thus, we use $T=4033$ log-returns for each market. These samples cover more than 15 years and encompass several world crises, such as the Asian crisis in 1997. The descriptive statistics of these data sets are provided in Table 5 in the Appendix. In the next subsections, we first investigate the probability distribution function for each data set, and then for each portfolio composed by two indexes. In a second step, we adjust several parametric models among those introduced in Section 2.

\subsection{Non-parametric model}

To establish the variability of the unconditional distribution of each return, we apply the concept of moving window. Then, on each moving window of 522 observations, we choose the best marginal distributions for each return among three probability density functions (pdf): the Gaussian pdf, the logistic pdf and the Laplace pdf. The 522-rolling window corresponds roughly to two years and we move this window every six months (130 points). 
We use the maximum likelihood method and the Akaike Information Criteria (AIC) to determine the best adjustment. Finally, we estimate $28 \mathrm{pdf}$ for each return. According to the AIC, the Laplace pdf is retained in most of the cases, with parameters evolving over the period. This confirms the fact that some non-stationarity characterizes the data sets which convinces us to study the $\mathrm{VaR}$ and ES measures in a dynamic way. Now, we compute the dynamic copula associated to each pair of markets $\left(\left(r_{i, t}\right)_{t},\left(r_{j, t}\right)_{t}\right), i, j=1,2,3$ by using the previous rolling windows.

In order to take into account the existence of co-movements in the markets we consider a set of copulas with different tail dependence behaviors. We retain the Gaussian $\left(C_{\text {Gau }}\right)$, Student- $t\left(C_{t}\right)$, Gumbel $\left(C_{G}\right)$, survival ${ }^{4}$ Gumbel $\left(C_{S G}\right)$, Clayton $\left(C_{C}\right)$, survival Clayton $\left(C_{S C}\right)$, Joe $\left(C_{J}\right)$, Survival Joe $\left(C_{S J}\right)$, Frank $\left(C_{F}\right)$ and Ali-Mikhail-Haq copulas $\left(C_{A}\right)$, Joe (1997). The Gaussian and Student- $t$ copulas are symmetric and belong to the Elliptical family. For the Student- $t$ copula, the parameter $\theta$ is a vector: $\theta=(\rho, v)$, where $\rho$ is the correlation coefficient and $v$ the number of degrees of freedom. The other copulas belong to the Archimedean class and are characterized by their generator function, Nelsen (1999). In order to use copulas which have upper and lower tail dependence without being symmetric, we define new copulas using a convex linear combination of two copulas. Hence, for $\omega \in[0,1]$ and two Archimedean copulas $C_{\theta_{1}}$ and $C_{\theta_{2}}$ we consider the copula $C$ such that:

$$
C(u, v)=\omega C_{\theta_{1}}(u, v)+(1-\omega) C_{\theta_{2}}(u, v) .
$$

The tail dependence parameters of these copulas can be derived from those of $C_{\theta_{1}}$ and $C_{\theta_{2}}$. In the remainder of the paper, several convex linear combinations are used. The Gumbel + Survival Gumbel $\left(C_{G S G}\right)$, Survival Clayton + Survival Gumbel $\left(C_{S C S G}\right)$, Survival Clayton + Clayton $\left(C_{S C C}\right)$, Gumbel + Clayton $\left(C_{G C}\right)$, Joe + Survival Joe $\left(C_{J S J}\right)$, Joe + Clayton $\left(C_{J C}\right)$ and Gumbel + Survival Joe $\left(C_{G S J}\right)$ copulas. By convention, the first copula has an upper tail dependence and the second one has a lower tail dependence. We denote again $\theta=\left(\theta_{1}, \theta_{2}\right)$ the vector parameter which characterizes these copulas.

\footnotetext{
${ }^{4}$ A survival copula $C_{S}$ of a copula $C$ is defined as follows: $C_{S}(u, v)=u+v-1+C(1-u$, $1-v)$.
} 
To estimate the parameter $\theta$ we use the pseudo log-likelihood function, Oakes (1994). Thus, using a sample of T observations, the pseudomaximum estimate $\hat{\theta}$ satisfies:

$$
\hat{\theta}=\underset{\theta}{\arg \max } \sum_{i=1}^{T} \log c\left(\hat{F}_{i T}\left(z_{i t}\right), \hat{F}_{j T}\left(z_{j t}\right)\right),
$$

where $c(u, v)=\frac{\partial^{2}}{\partial u \partial v} C_{\theta}(u, v)$ and

$$
\hat{F}_{i T}\left(z_{i T}\right)=\frac{1}{T+1} \sum_{p=1}^{T} 1_{\left\{z_{i p}<z_{i i}\right\}}, i=1,2,3 .
$$

This last quantity represents the empirical marginal distribution for each market. Using the pseudo-maximum likelihood method permits us to get an AIC value that we use to discriminate between the different copulas. We retain the copula for which the AIC value is the smallest.

In Table 1, we provide the selected copulas (using AIC) with the parameters' estimates, for each window and each pair of markets. We omitted the standard error of the estimates but this statistic can be calculated using a Jacknife method. Notice that, in Caillault and Guégan (2005), using the whole sample, we have found a Student- $t$ copula for the pairs $\left(\left(r_{1, t}\right)_{t},\left(r_{2, t}\right)_{t}\right)$ and $\left(\left(r_{2, t}\right)_{t},\left(r_{3, t}\right)_{t}\right)$ and a $C_{G S G}$ copula for the pair $\left(\left(r_{1, t}\right)_{t},\left(r_{3, t}\right)_{t}\right)$. Here, the selected copulas are generally tail dependent. The Gaussian copula is selected for the pairs $\left(\left(r_{1, t}\right)_{t},\left(r_{2, t}\right)_{t}\right)$ and $\left(\left(r_{2, t}\right)_{t},\left(r_{3, t}\right)_{t}\right)$, the Frank copula is selected for the pairs $\left(\left(r_{2, t}\right)_{t},\left(r_{3, t}\right)_{t}\right)$ and $\left(\left(r_{1, t}\right)_{t},\left(r_{3, t}\right)_{t}\right)$ and the Ali-Mikhail-Haq copula for the pair $\left(\left(r_{1, t}\right)_{t},\left(r_{3, t}\right)_{t}\right)$. This adjustment means that the markets appear independent in the tails and this for the periods on which the Gaussian, Frank and Ali Mikhail Haq copulas are adjusted.

For the pair $\left(\left(r_{1, t}\right)_{t},\left(r_{2, t}\right)_{t}\right)$ we selected seven copulas, ten copulas for the pair $\left(\left(r_{1, t}\right)_{t},\left(r_{3, t}\right)_{t}\right)$ and eight copulas for the pair $\left(\left(r_{2, t}\right)_{t},\left(r_{3, t}\right)_{t}\right)$. Thus, the dependence structure is not the same for the whole period under study. The 
copulas and also their parameters change through time. For instance, if we considered the pair $\left(\left(r_{2, t}\right)_{t},\left(r_{3, t}\right)_{t}\right)$, between 1995 and 1998, the parameter $\rho$ of the Student- $t$ copula is stable whereas the parameter $v$ changes. This implies an important change in the tail behavior of the distribution. For this pair of markets, we observe that between December 23, 1996, the lower tail dependent parameter grows from $\lambda_{L}=2 \bar{t}_{5.66+1}\left(\sqrt{\frac{(5.66+1)(1-0.58)}{1+0.58}}\right)=0.23$ to $\lambda_{L}=2 \bar{t}_{2.00+1}\left(\sqrt{\frac{(2.00+1)(1-0.54)}{1+0.54}}\right)=0.41 \quad$ on June $22,1998 \quad$ (where $\bar{t}_{v+1}(x)=1-t_{v+1}(x)$ is the univariate student- $t$ distribution with $v+1$ degrees of freedom). Thus, during this period, a co-movement exists between the Malaysian and Indonesian markets. The results concerning the other pairs of markets confirm this existence of non-stationarity for the bivariate distribution.

Table 1 Estimation of copulas, using the moving window, for each pair of markets.

\begin{tabular}{|c|c|c|c|c|c|c|c|c|c|c|c|c|}
\hline & \multicolumn{4}{|c|}{ Thai/Mal } & \multicolumn{4}{|c|}{ Thai//Indo } & \multicolumn{4}{|c|}{$\mathrm{Mal} /$ Indo } \\
\hline Dates & Copulas & & Paramete & & Copulas & & Paramete & & Copulas & & Paramete & \\
\hline 03/07/1989 & Ct & 0.47 & 3.44 & - & CGSG & 2.20 & 1.00 & 0.50 & CGSG & 2.58 & 1.06 & 0.34 \\
\hline 01/01/1990 & $\mathrm{Ct}$ & 0.55 & 4.00 & - & $\mathrm{Ct}$ & 0.36 & 3.88 & - & CGSG & 2.26 & 1.04 & 0.42 \\
\hline 02/07/1990 & $\mathrm{Ct}$ & 0.47 & 5.95 & - & CscsG & 0.04 & 2.45 & 0.64 & CGSG & 1.04 & 3.01 & 0.63 \\
\hline $31 / 12 / 1990$ & $\mathrm{Ct}$ & 0.46 & 4.05 & - & CF & 2.58 & - & - & CGSG & 3.14 & 1.13 & 0.32 \\
\hline 01/07/1991 & CGSG & 1.17 & 1.75 & 0.39 & CF & 2.51 & - & - & CGSG & 2.93 & 1.16 & 0.28 \\
\hline $30 / 12 / 1991$ & $\mathrm{Ct}$ & 0.42 & 4.71 & - & CF & 2.26 & - & - & $\mathrm{Ct}$ & 0.40 & 4.26 & - \\
\hline 29/06/1992 & CscsG & 0.25 & 1.78 & 0.42 & $\mathrm{Ct}$ & 0.31 & 5.29 & - & $\mathrm{Ct}$ & 0.39 & 5.46 & - \\
\hline 28/12/1992 & $\mathrm{Ct}$ & 0.43 & 7.25 & 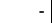 & $\mathrm{Ct}$ & 0.31 & 5.23 & - & $\mathrm{CF}_{\mathrm{F}}$ & 2.74 & - & - \\
\hline 28/06/1993 & $\mathrm{Ct}$ & 0.35 & 6.36 & - & CscsG & 10.74 & 1.20 & 0.06 & CF & 2.91 & - & - \\
\hline $27 / 12 / 1993$ & $\mathrm{Ct}$ & 0.42 & 9.21 & - & $\mathrm{CA}$ & 0.79 & - & - & CGC & 2.00 & 0.47 & 0.37 \\
\hline $27 / 06 / 1994$ & CJC & 1.53 & 1.22 & 0.43 & $\mathrm{CA}$ & 0.85 & - & - & CGC & 2.73 & 0.47 & 0.24 \\
\hline $26 / 12 / 1994$ & $\mathrm{Ct}$ & 0.48 & 3.89 & 1 & CGSG & 1.00 & 1.72 & 0.35 & CGC & 2.57 & 0.51 & 0.22 \\
\hline $26 / 06 / 1995$ & CGSG & 1.00 & 1.85 & 0.15 & CscsG & - & 1.75 & 0.23 & $\mathrm{CGC}_{\mathrm{GC}}$ & 2.08 & 0.68 & 0.26 \\
\hline $25 / 12 / 1995$ & $\mathrm{Ct}$ & 0.57 & 3.50 & - & $\mathrm{Ct}$ & 0.53 & 3.46 & 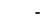 & $\mathrm{Ct}$ & 0.53 & 7.58 & - \\
\hline $24 / 06 / 1996$ & $\mathrm{Ct}$ & 0.57 & 5.22 & - & $\mathrm{Ct}$ & 0.59 & 4.22 & - & $\mathrm{Ct}$ & 0.58 & 6.72 & - \\
\hline $23 / 12 / 1996$ & $\mathrm{Ct}$ & 0.55 & 6.63 & - & $\mathrm{Ct}$ & 0.56 & 7.08 & - & $\mathrm{Ct}$ & 0.58 & 5.66 & - \\
\hline 23/06/1997 & $\mathrm{Ct}$ & 0.45 & 5.21 & - & $\mathrm{Ct}$ & 0.45 & 10.18 & - & CJC & 1.83 & 1.35 & 0.43 \\
\hline 22/12/1997 & CGSG & 1.05 & 2.58 & 0.58 & $\mathrm{Ct}$ & 0.42 & 3.91 & 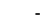 & $\mathrm{Ct}$ & 0.54 & 2.33 & - \\
\hline 22/06/1998 & $\mathrm{Ct}$ & 0.46 & 3.35 & ( & CGSJ & 2.35 & 1.21 & 0.35 & $\mathrm{Ct}$ & 0.54 & 2.00 & - \\
\hline $21 / 12 / 1998$ & CGSJ & 1.43 & 2.01 & 0.70 & CGSJ & 1.90 & 1.15 & 0.49 & CGSJ & 2.61 & 1.35 & 0.39 \\
\hline 21/06/1999 & CGau & 0.49 & - & 1 & CJSJ & 2.06 & 1.43 & 0.45 & $\mathrm{Ct}$ & 0.42 & 5.00 & - \\
\hline 20/12/1999 & CscsG & 2.96 & 1.37 & 0.19 & CJSJ & 2.00 & 1.39 & 0.48 & $\mathrm{Ct}$ & 0.36 & 4.40 & - \\
\hline $19 / 06 / 2000$ & CscsG & 4.39 & 1.24 & 0.11 & CG & 1.31 & - & - & $\mathrm{Ct}$ & 0.26 & 9.05 & - \\
\hline $18 / 12 / 2000$ & CsG & 1.28 & - & . & CGau & 0.34 & - & - & CsG & 1.14 & - & - \\
\hline $18 / 06 / 2001$ & CSG & 1.27 & - & - & $\mathrm{Cc}$ & 0.38 & - & - & CsJ & 1.24 & - & - \\
\hline $17 / 12 / 2001$ & CsG & 1.31 & - & - & $\mathrm{Cc}$ & 0.34 & - & - & CSG & 1.19 & - & - \\
\hline $17 / 06 / 2002$ & CSG & 1.32 & - & 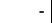 & CGau & 0.26 & - & - & CSG & 1.19 & - & - \\
\hline $17 / 12 / 2002$ & CsG & 1.39 & - & - & CA & 0.70 & - & - & CSG & 1.26 & - & - \\
\hline
\end{tabular}

\subsection{Parametric models}

In order to take into account the volatility of the log-returns that we have observed on each return (see table 5 in the Annex), we adjusted, on each 
pair of markets, several GARCH models denoted DVEC(1,1), MDIAG(1,1), $\operatorname{BEKK}(1,1)$, DVEC- $t(1,1)$, MDIAG- $t(1,1)$, BEKK- $t(1,1)$ whose expressions are given in Section 2.1.2. At the end, we have retained the $\operatorname{DVEC}(1,1)$ and the DVEC- $t(1,1)$ for all pairs of markets according to the smallest values of AIC. All the parameters are significant.

Because jumps have been observed inside the data sets, we also select two Markov switching models with two or three regimes and an AR(1) model on each regime. We denote them MSIH and MSIH-3r. These models correspond to the best models according to the Hannan Quinn, Schwartz and AIC criteria.

\section{4 - VaR and ES measures for Asian portfolios}

Now, we use the previous models to compute the VaR and ES measures for the three Asian portfolios. We denote these portfolios respectively $P_{1}, P_{2}$ and $P_{3}$. They are equally weighted. The $\mathrm{VaR}$ and ES measures are calculated for $\alpha=1 \%$. Using the empirical histogram and the copula approaches we compute $28 \mathrm{VaR}$ and ES values. With the RiskMetrics, GARCH and Markov switching models, the VaR and ES measures are computed for all $t=1, \ldots, T$. We provide the values corresponding to the 28 dates retained with the two other methods. The first VaR measure is calculated at date July 3, 1989 and the last one at date December 17, 2002.

In Table 2, we reported the values for the dynamic VaR measure computed with seven models for the portfolio $P_{l}$. We plotted on Figure 1 the trajectories of the VaR measure for the portfolio $P_{l}$. The black dashed line represents the $\mathrm{VaR}$ measure computed on the whole period using the empirical histogram approach. It is equal to -5.03\%. All the trajectories are far from this constant value which justifies working in a dynamic framework. On Figure 1, we have in 1991, (2) The Mexican Tequila crisis in 1994-1995, (3) The Asian crisis in 1997, (4) the Russian crisis in 1998, (5) the bursting of the technological speculative bubble in 2000. The Asian and Russian crises are presented on the same vertical rectangle.

On Figure 1, we observe similar shapes for the evolution of the VaR measure whatever the method used. Note that we have the same behavior for the portfolios $P_{2}$ and $P_{3}$ (they can be provided on request). The copulas and the empirical histogram approaches give similar results. 
Cyril Caillault, Dominique Guégan - Forecasting VaR and Expected Shortfall using Dynamical Systems: A Risk Management Strategy - Frontiers in Finance and Economics - Vol. 6 No.1 - April 2009,

Notice that these two last methods are based on the unconditional distribution of the portfolios. Nevertheless, the empirical histogram method provides VaR values which exhibit jumps followed by plateaus, for instance for the portfolio $P_{l}$ from December 31, 1990 to June 29, 1993. This behavior appears when negative returns stay in the window during several sub-periods. The copula approach avoids this unpleasant effect. In most of the cases, the VaR values obtained with the empirical histogram method are lower than those obtained with the copula method.

Table 2 Values of the dynamic VaR calculated with the seven approaches.

\begin{tabular}{|c|c|c|c|c|c|c|c|}
\hline & \multicolumn{7}{|c|}{ Thai/Mal } \\
\hline Date & Copula & $\overline{\mathrm{EH}}$ & $\overline{\mathrm{RM}}$ & DVEC & DVEC- $t$ & $\mathrm{MSIH}$ & MSIH-3r \\
\hline $03 / 07 / 1989$ & $-3.56 \%$ & $-6.68 \%$ & $-3.74 \%$ & $-3.46 \%$ & $-3.87 \%$ & $-2.72 \%$ & $-2.82 \%$ \\
\hline $01 / 01 / 1990$ & $-2.81 \%$ & $-3.00 \%$ & $-2.33 \%$ & $-2.58 \%$ & $-2.89 \%$ & $-2.34 \%$ & $-2.17 \%$ \\
\hline $02 / 07 / 1990$ & $-2.92 \%$ & $-2.62 \%$ & $-2.17 \%$ & $-2.56 \%$ & $-2.87 \%$ & $-2.40 \%$ & $-2.27 \%$ \\
\hline $31 / 12 / 1990$ & $-3.65 \%$ & $-4.46 \%$ & $-3.34 \%$ & $-3.10 \%$ & $-3.47 \%$ & $-2.55 \%$ & $-2.88 \%$ \\
\hline 01/07/1991 & $-4.16 \%$ & $-4.46 \%$ & $-2.27 \%$ & $-2.77 \%$ & $-3.10 \%$ & $-2.33 \%$ & $-2.16 \%$ \\
\hline $30 / 12 / 1991$ & $-4.15 \%$ & $-4.46 \%$ & $-2.08 \%$ & $-2.41 \%$ & $-2.69 \%$ & $-2.36 \%$ & $-2.19 \%$ \\
\hline 29/06/1992 & $-3.93 \%$ & $-4.52 \%$ & $-2.91 \%$ & $-2.57 \%$ & $-2.88 \%$ & $-2.37 \%$ & $-2.20 \%$ \\
\hline 28/12/1992 & $-3.34 \%$ & $-3.31 \%$ & $-2.09 \%$ & $-2.39 \%$ & $-2.67 \%$ & $-2.34 \%$ & $-2.14 \%$ \\
\hline 28/06/1993 & $-2.71 \%$ & $-3.00 \%$ & $-2.85 \%$ & $-3.48 \%$ & $-3.89 \%$ & $-6.48 \%$ & $-4.57 \%$ \\
\hline $27 / 12 / 1993$ & $-2.38 \%$ & $-2.72 \%$ & $-3.34 \%$ & $-3.20 \%$ & $-3.57 \%$ & $-3.42 \%$ & $-3.26 \%$ \\
\hline $27 / 06 / 1994$ & $-3.09 \%$ & $-4.23 \%$ & $-3.06 \%$ & $-3.28 \%$ & $-3.67 \%$ & $-4.40 \%$ & $-4.29 \%$ \\
\hline $26 / 12 / 1994$ & $-3.36 \%$ & $-4.23 \%$ & $-2.73 \%$ & $-3.01 \%$ & $-3.36 \%$ & $-2.34 \%$ & $-2.17 \%$ \\
\hline $26 / 06 / 1995$ & $-4.22 \%$ & $-4.49 \%$ & $-2.58 \%$ & $-2.48 \%$ & $-2.77 \%$ & $-2.35 \%$ & $-2.16 \%$ \\
\hline 25/12/1995 & $-4.24 \%$ & $-4.49 \%$ & $-2.11 \%$ & $-2.57 \%$ & $-2.87 \%$ & $-2.34 \%$ & $-2.18 \%$ \\
\hline $24 / 06 / 1996$ & $-3.04 \%$ & $-3.20 \%$ & $-1.62 \%$ & $-2.30 \%$ & $-2.57 \%$ & $-2.37 \%$ & $-2.21 \%$ \\
\hline $23 / 12 / 1996$ & $-3.21 \%$ & $-3.20 \%$ & $-2.18 \%$ & $-2.67 \%$ & $-2.98 \%$ & $-2.39 \%$ & $-2.57 \%$ \\
\hline $23 / 06 / 1997$ & $-2.96 \%$ & $-3.05 \%$ & $-4.34 \%$ & $-4.56 \%$ & $-5.10 \%$ & $-7.37 \%$ & $-12.02 \%$ \\
\hline 22/12/1997 & $-4.90 \%$ & $-5.32 \%$ & $-9.86 \%$ & $-9.27 \%$ & $-10.36 \%$ & $-7.20 \%$ & $-8.16 \%$ \\
\hline $22 / 06 / 1998$ & $-7.21 \%$ & $-7.89 \%$ & $-8.47 \%$ & $-8.74 \%$ & $-9.77 \%$ & $-7.36 \%$ & $-10.27 \%$ \\
\hline 21/12/1998 & $-7.97 \%$ & $-8.34 \%$ & $-4.61 \%$ & $-3.76 \%$ & $-4.20 \%$ & $-2.40 \%$ & $-3.17 \%$ \\
\hline 21/06/1999 & $-8.00 \%$ & $-8.34 \%$ & $-4.22 \%$ & $-3.38 \%$ & $-3.78 \%$ & $-7.10 \%$ & $-4.97 \%$ \\
\hline 20/12/1999 & $-7.89 \%$ & $-6.16 \%$ & $-3.17 \%$ & $-3.21 \%$ & $-3.59 \%$ & $-2.47 \%$ & $-2.61 \%$ \\
\hline $19 / 06 / 2000$ & $-5.48 \%$ & $-4.96 \%$ & $-3.80 \%$ & $-3.53 \%$ & $-3.95 \%$ & $-7.16 \%$ & $-4.69 \%$ \\
\hline $18 / 12 / 2000$ & $-4.81 \%$ & $-4.04 \%$ & $-1.98 \%$ & $-2.43 \%$ & $-2.71 \%$ & $-2.60 \%$ & $-2.86 \%$ \\
\hline $18 / 06 / 2001$ & $-4.21 \%$ & $-4.04 \%$ & $-2.73 \%$ & $-3.06 \%$ & $-3.43 \%$ & $-2.44 \%$ & $-3.11 \%$ \\
\hline $17 / 12 / 2001$ & $-3.81 \%$ & $-4.46 \%$ & $-2.51 \%$ & $-2.71 \%$ & $-3.03 \%$ & $-2.33 \%$ & $-2.16 \%$ \\
\hline $17 / 06 / 2002$ & $-3.24 \%$ & $-3.31 \%$ & $-1.89 \%$ & $-2.51 \%$ & $-2.80 \%$ & $-2.35 \%$ & $-2.19 \%$ \\
\hline $17 / 12 / 2002$ & $-2.94 \%$ & $-3.76 \%$ & $-2.32 \%$ & $-2.97 \%$ & $-3.32 \%$ & $-2.37 \%$ & $-2.35 \%$ \\
\hline
\end{tabular}

Values of the dynamic $\mathrm{VaR}$ measure for the portfolio $\mathrm{P}_{1}$ at $99 \%$ confidence level over a time interval from July 3, 1989 to December 17, 2002.

The values of the VaR measure computed using the RiskMetrics, DVEC, DVEC- $t$ models are close to each other. With the DVEC- $t$ model, we 
got the smallest VaR values. This is due to the tail behavior of the $t$ distribution. The empirical histogram and the copula approaches provide lower $\mathrm{VaR}$ measures than those obtained using the previous models, except during The Asian and Russian crises.

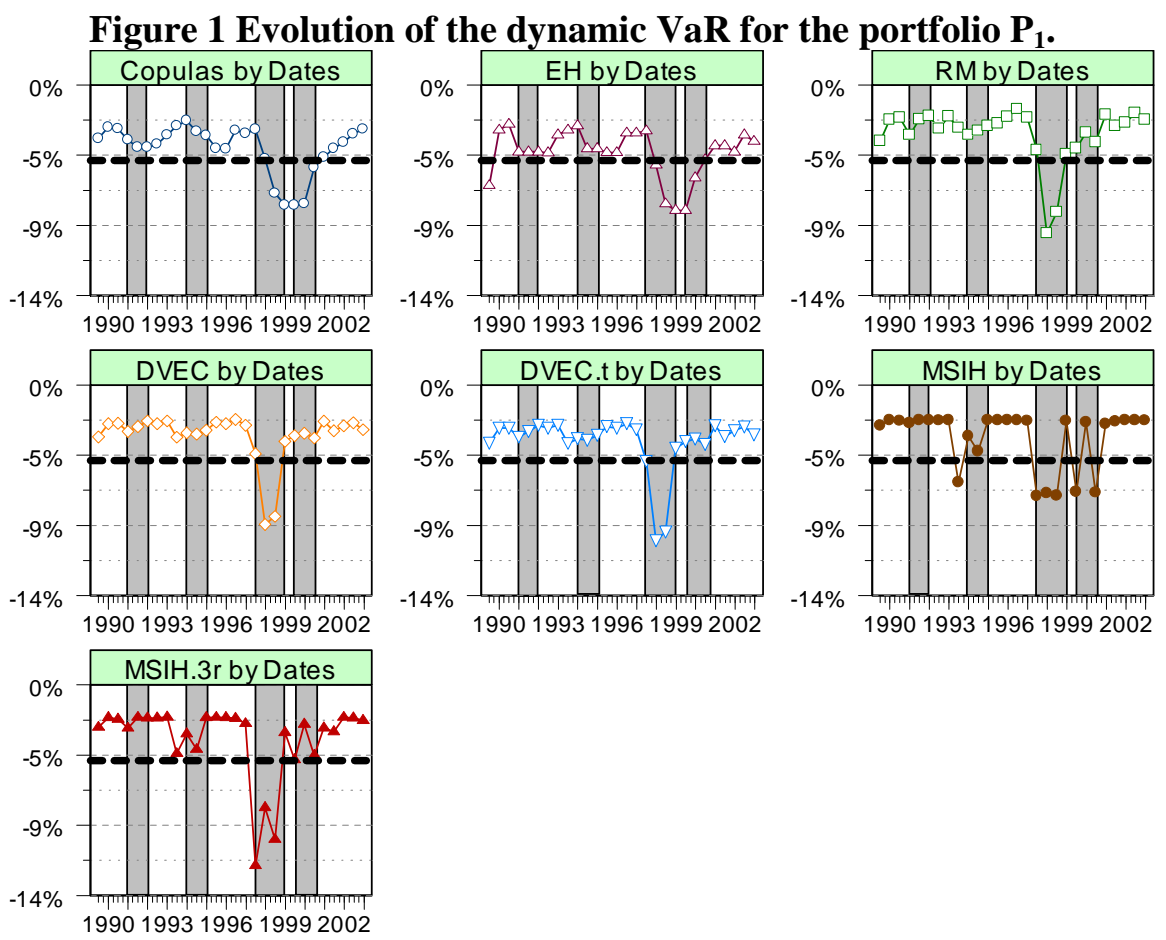

Values of the dynamic VaR measure for the portfolio $P_{l}$ at $99 \%$ confidence level over a time interval from July 3, 1989 to December 17, 2002. The VaR measures are represented by a circle for the dynamic copulas' approach, by a triangle for the empirical histogram approach and so on. The black dash line represents the VaR measure computed on the whole period using the empirical histogram approach and is equal to $-5.03 \%$. The vertical rectangles represent the different crises over the period. 
The VaR values computed with the Markov switching models depend on the estimated probability transition matrix and on the filtered probabilities which, here, are close to zero or one. Thus, the values of the VaR measure are stable inside a given regime. They are larger than the values obtained with all the previous methods, except for some dates that we discuss below.

Now, we specify the impact of the crisis on the evolution of the three portfolios.

- The portfolio $P_{1}$ is affected by The first Gulf war and The Mexican Tequila crisis. Using the copula and the empirical histogram approaches, we see that the VaR values obtained for this portfolio change as soon as the two crises appear. On the other hand, the stochastic volatility models do not take into account these two crises and the Markov switching models are unaware concerning the crisis provoked by the first Gulf war. The $\mathrm{VaR}$ values obtained for this portfolio, with the MSIH-3r model, are four times the VaR computed with the copula method (-12.02\% against $-2.96 \%)$.

- For the portfolios $P_{1}$ and $P_{2}$, the Markov switching approach provides small VaR values just before the Asian crisis at date June 23, 1997 (officially the Asian crisis started in July, 1997). We observe that the Markov switching models overestimate the risk of these portfolios at this date when we compare their results with the values obtained from the other models.

- For the three portfolios, at date June 28, 1993, we observe a peak using the Markov switching approach between the first Gulf war and the Mexican Tequila crises. This peak does not appear with the other models. The three portfolios are dramatically affected by the Asian and Russian crises. During these two periods the seven models provide their lowest VaR values. At time of the bursting of the technological speculative bubble in 2000 the values of the $\mathrm{VaR}$ measure stay low except for those obtained with the GARCH models. But this crisis is less significant than the Asian one.

The values of the dynamic ES measure obtained for the portfolio $P_{l}$, with the seven models are provided in Table 3. The Figure 2 represents the values of the dynamic ES measure for this portfolio. The black dash line represents the constant ES measure computed on the whole period using the 
Cyril Caillault, Dominique Guégan - Forecasting VaR and Expected Shortfall using Dynamical Systems: A Risk Management Strategy - Frontiers in Finance and Economics - Vol. 6 No.1 - April 2009,

empirical histogram approach. It is equal to $-7.08 \%$. The shapes that we obtained are similar whatever the methods used. We can summarize the results in the following way:

- The parametric models give in mean lower ES value than the copula method (and not only during the Asian and Russian crises).

- For the portfolios $P_{2}$ and $P_{3}$, on December 22, 1997, the ES measures are identical with the Riskmetrics and GARCH models. This comes from the lack of observations.

- For the portfolio $P_{l}$, the MSIH model does not react to the first Gulf war crisis at the level of difference of all other models that take into account all the crises.

Table 3 Values of the dynamic Expected Shortfall calculated with the seven approaches.

\begin{tabular}{|r|ccccccc|}
\cline { 2 - 7 } \multicolumn{1}{c|}{} & \multicolumn{7}{c|}{ Thai/Mal } \\
\hline Date & Copula & EH & RM & DVEC & DVEC- & MSIH & MSIH-3r \\
\hline $01 / 07 / 1989$ & $-5.61 \%$ & $-8.54 \%$ & $-6.45 \%$ & $-6.25 \%$ & $-6.45 \%$ & $-5.30 \%$ & $-5.30 \%$ \\
$02 / 1990$ & $-3.41 \%$ & $-5.35 \%$ & $-4.80 \%$ & $-5.09 \%$ & $-5.67 \%$ & $-4.80 \%$ & $-4.55 \%$ \\
$31 / 12 / 1990$ & $-3.49 \%$ & $-4.16 \%$ & $-4.49 \%$ & $-5.09 \%$ & $-5.67 \%$ & $-4.99 \%$ & $-4.64 \%$ \\
$01 / 07 / 1991$ & $-5.13 \%$ & $-7.81 \%$ & $-6.33 \%$ & $-6.20 \%$ & $-6.46 \%$ & $-5.07 \%$ & $-5.85 \%$ \\
$30 / 12 / 1991$ & $-5.12 \%$ & $-7.81 \%$ & $-4.32 \%$ & $-5.20 \%$ & $-5.89 \%$ & $-4.40 \%$ & $-4.17 \%$ \\
$29 / 06 / 1992$ & $-5.20 \%$ & $-7.01 \%$ & $-3.96 \%$ & $-4.42 \%$ & $-4.92 \%$ & $-4.31 \%$ & $-4.11 \%$ \\
$28 / 12 / 1992$ & $-4.05 \%$ & $-3.92 \%$ & $-3.30 \%$ & $-4.59 \%$ & $-5.30 \%$ & $-4.24 \%$ & $-4.09 \%$ \\
$28 / 06 / 1993$ & $-3.40 \%$ & $-3.81 \%$ & $-5.11 \%$ & $-4.24 \%$ & $-4.67 \%$ & $-4.18 \%$ & $-3.91 \%$ \\
$27 / 12 / 1993$ & $-2.93 \%$ & $-3.30 \%$ & $-5.92 \%$ & $-5.67 \%$ & $-6.57 \%$ & $-8.88 \%$ & $-7.88 \%$ \\
$27 / 06 / 1994$ & $-3.59 \%$ & $-5.64 \%$ & $-5.39 \%$ & $-5.50 \%$ & $-5.92 \%$ & $-6.12 \%$ & $-5.67 \%$ \\
$26 / 12 / 1994$ & $-4.06 \%$ & $-5.64 \%$ & $-4.78 \%$ & $-5.30 \%$ & $-5.56 \%$ & $-4.14 \%$ & $-6.85 \%$ \\
$26 / 06 / 1995$ & $-5.55 \%$ & $-5.74 \%$ & $-4.42 \%$ & $-4.23 \%$ & $-4.70 \%$ & $-4.06 \%$ & $-3.85 \%$ \\
$25 / 12 / 1995$ & $-5.41 \%$ & $-5.74 \%$ & $-3.71 \%$ & $-4.36 \%$ & $-5.02 \%$ & $-4.03 \%$ & $-3.83 \%$ \\
$24 / 06 / 1996$ & $-3.98 \%$ & $-3.78 \%$ & $-2.98 \%$ & $-3.98 \%$ & $-4.32 \%$ & $-4.04 \%$ & $-3.85 \%$ \\
$23 / 12 / 1996$ & $-4.08 \%$ & $-3.82 \%$ & $-3.80 \%$ & $-4.41 \%$ & $-5.01 \%$ & $-4.04 \%$ & $-4.34 \%$ \\
$23 / 06 / 1997$ & $-3.87 \%$ & $-3.64 \%$ & $-6.77 \%$ & $-7.38 \%$ & $-7.85 \%$ & $-9.73 \%$ & $-12.94 \%$ \\
$22 / 12 / 1997$ & $-6.36 \%$ & $-7.23 \%$ & $-11.48 \%$ & $-11.48 \%$ & $-12.12 \%$ & $-9.00 \%$ & $-9.74 \%$ \\
$22 / 06 / 1998$ & $-9.35 \%$ & $-9.15 \%$ & $-9.91 \%$ & $-10.46 \%$ & $-11.52 \%$ & $-9.46 \%$ & $-11.97 \%$ \\
$21 / 12 / 1998$ & $-10.09 \%$ & $-10.08 \%$ & $-6.83 \%$ & $-5.69 \%$ & $-6.27 \%$ & $-4.20 \%$ & $-5.11 \%$ \\
$21 / 06 / 1999$ & $-10.23 \%$ & $-10.08 \%$ & $-6.26 \%$ & $-5.22 \%$ & $-5.66 \%$ & $-9.32 \%$ & $-7.31 \%$ \\
$20 / 12 / 1999$ & $-10.28 \%$ & $-9.32 \%$ & $-4.97 \%$ & $-4.99 \%$ & $-5.35 \%$ & $-4.20 \%$ & $-4.37 \%$ \\
$19 / 06 / 2000$ & $-7.13 \%$ & $-6.87 \%$ & $-5.63 \%$ & $-5.24 \%$ & $-5.80 \%$ & $-9.46 \%$ & $-6.83 \%$ \\
$18 / 12 / 2000$ & $-5.98 \%$ & $-4.72 \%$ & $-3.51 \%$ & $-4.07 \%$ & $-4.43 \%$ & $-4.32 \%$ & $-4.67 \%$ \\
$18 / 06 / 2001$ & $-4.97 \%$ & $-4.62 \%$ & $-4.46 \%$ & $-4.86 \%$ & $-5.17 \%$ & $-4.08 \%$ & $-4.93 \%$ \\
$17 / 12 / 2001$ & $-5.35 \%$ & $-5.41 \%$ & $-4.19 \%$ & $-4.43 \%$ & $-4.83 \%$ & $-3.99 \%$ & $-3.78 \%$ \\
$17 / 06 / 2002$ & $-4.23 \%$ & $-4.87 \%$ & $-3.41 \%$ & $-4.19 \%$ & $-4.60 \%$ & $-3.99 \%$ & $-3.81 \%$ \\
$17 / 12 / 2002$ & $-4.17 \%$ & $-4.95 \%$ & $-3.94 \%$ & $-4.75 \%$ & $-5.04 \%$ & $-4.00 \%$ & $-3.98 \%$ \\
\hline
\end{tabular}

Values of the dynamic Expected Shortfall measure for the portfolio $\mathrm{P}_{1}$ at $99 \%$ confidence level over a time interval from July 3, 1989 to December 17, 2002. 
Figure 2 Evolution of the dynamic ES for the portfolio $P_{1}$.
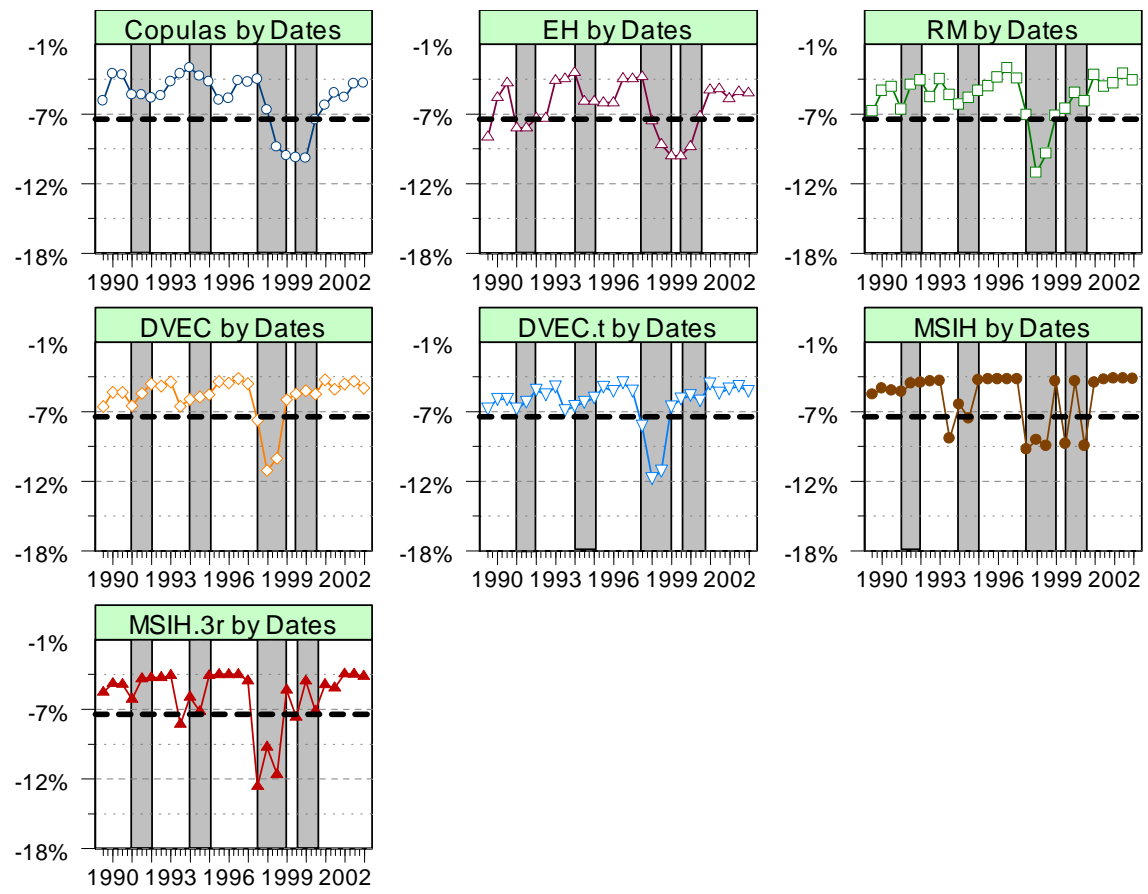

Values of the dynamic ES measure for the portfolio $\mathrm{P}_{1}$ at $99 \%$ confidence level over a time interval from July 3, 1989 to December 17, 2002. The ES measures are represented by a circle for the dynamic copulas' approach, by a triangle for the empirical histogram approach and so on. The black dash line represents the ES measure computed on the whole period using the empirical histogram approach and is equal to $-7.08 \%$. The vertical rectangles represent the different crises through the period.

Thus we can observe that the values we obtained for the market risk with the VaR and ES measures are different. According to the equation (2), the ES measure is always a minorant of the VaR measure for the 28 dates we considered. For instance for the portfolio $P_{1}$ with the copula approach, in December 22, 1997, the value of the VaR measure is $-4.90 \%$ and the value of the ES measure is $-6.36 \%$. Note that the market risk computed with ES 
measure is very low for the three portfolios. For instance, for the portfolio $P_{3}$, using the DVEC- $t$ model, we get ES $=-18.22 \%$ on December 22,1997 , which is a very low value. In that case, the ES measure is more significant than the $\mathrm{VaR}$ measure. This means that the VaR measure, in some cases, can be unable to take into account high negative returns. The Bis committee of the Global Market in 1999 justifies the non-anticipation of the financial crisis in autumn 1998 by the fact that one uses the VaR. Indeed, this measure can, in certain cases, be inefficient and it seems interesting to use the ES measure which is more informative by taking into account the behavior of the portfolios' tail distributions.

\section{5- Conclusion}

In this paper, we use several models to compute the Value at Risk and the Expected Shortfall measures for Asian portfolios composed of two indexes. Under the constraint of validation by the regulators, banks are free to choose one of the models developed here to answer the requirement stated by the 1996 Basle amendment. This amendment stipulates that banks have to develop their own internal models to compute a measure of market risk every day and have to test their accuracy over a period of 250 business days.

On the basis of this rule, we have investigated the dynamics of three portfolios via their distribution. We show, using the copula approach and parametric models that the bivariate distribution of a portfolio is not stable over the period under study. This is the reason why we compute the Value at Risk and the Expected Shortfall measures in a dynamic way.

Another point of the Basle amendment concerns the computation of a capital requirement to cover the market risk. The effective daily capital requirement is the absolute value of the minimum of the previous day's $\mathrm{VaR}$ and $k$ times the average of the daily VaR over the last 60 days. The multiplier $k$ is a penalization coefficient ranging between 3 and 4 and depends on the backtesting results. The bigger the number of exceptions (the number of time that the model fails over the last 250 trading days), the more the supervisors increase $k$.

Our results show significant differences in the Value at Risk measures we obtained according to the method we used. This implies differences for the capital requirement value. It constitutes an opportunity for banks (which 
prefer to have a low capital requirement) to develop their internal VaR model. If we use this opportunity, the GARCH approach seems more appropriate (except during the Asian crisis). This choice shows that capital requirement managment can lead developing some market practices which are unsuitable. The copula approach appears more attractive for banks, because it permits them to take into account all crises, and does not require a large capital requirement during important crises such as the Asian one, periods for which the banks need liquidity.

Moreover, our results can be used to select the least risky of the three portfolios $P_{1}, P_{2}, P_{3}$. In practice, a risk averse manager invests in the portfolio for which the absolute Value at Risk measure is the smallest. The criteria we propose here is the following: we will select the portfolio for which the absolute Value at Risk and the Expected Shortfall measures are the smallest. We apply this criterion at five different dates during the crises: July 01, 1991, June 27, 1994, December 22, 1997, December 21, 1998 and December 18, 2000. For these five dates we have seven values for the Value at Risk and the Expected Shortfall measures. We apply the previous method at each date for the three portfolios. We reported the absolute value of the Value at Risk and Expected Shortfall measures in Table 4. Then, according to our previous criteria, we select the following best portfolios:

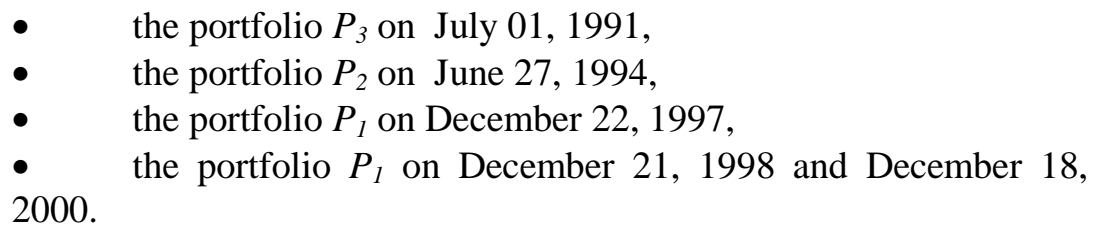

If we have only the choice between the portfolios $P_{1}$ and $P_{2}$ on July 01, 1991 the selection is more complicated. Indeed, using the Value at Risk measure, we select the portfolio $P_{l}$, but using the Expected Shortfall measure we select the portfolio $P_{2}$ because the extreme losses are bigger with $P_{1}$ than with $P_{2}$. In that latter case, the Expected Shortfall measure is more informative than the $\mathrm{VaR}$ measure. Thus, using these two measures seems important in a risk management strategy.

The question of forecasting, underlying this work, is of great interest. Historically, it seems natural to use the parametric models to forecast the VaR and the ES measures. Nevertheless this work shows that the use of the 
dynamic copula is interesting. Indeed, it permits, using the last period on which the model appears stationary, the use of the copula estimated on this sub-sample to forecast the $\mathrm{VaR}$ and the ES measures. The interest of the copula lies on the fact that the approach is free of assumptions. We just use the notion of local stationarity, whose interest has been recently discussed in Guégan $(2005,2007 a, b)$.

Table 4 Maximum absolute values of the VaR and ES measure at $99 \%$

\begin{tabular}{|ccccccc|}
\hline & & & \multicolumn{5}{c|}{ P1 } \\
\hline \multirow{2}{*}{ VaR 99\% } & Method & EH & MSIH & DVEC-t & EH & Copulas \\
& Value & $4.46 \%$ & $4.40 \%$ & $10.36 \%$ & $8.34 \%$ & $4.81 \%$ \\
& Method & EH & MSIH & DVEC-t & Copulas & Copulas \\
& Value & $7.81 \%$ & $7.22 \%$ & $12.12 \%$ & $10.09 \%$ & $5.98 \%$ \\
\hline VaR 99\% & Method & EH & DVEC-t & DVEC-t & EH & EH \\
& Value & $4.84 \%$ & $3.14 \%$ & $15.58 \%$ & $11.76 \%$ & $5.58 \%$ \\
ES 99\% & Method & EH & DVEC-t & DVEC-t & EH & EH \\
& Value & $6.48 \%$ & $4.46 \%$ & $17.02 \%$ & $13.57 \%$ & $6.72 \%$ \\
\hline & & & & P3 & & \\
\hline VaR 99\% & Method & EH & EH & DVEC-t & EH & EH \\
& Value & $3.90 \%$ & $3.34 \%$ & $16.87 \%$ & $12.83 \%$ & $4.99 \%$ \\
ES 99\% & Method & EH & DVEC-t & DVEC-t & EH & MSIH.3r \\
& Value & $5.83 \%$ & $5.28 \%$ & $18.22 \%$ & $15.71 \%$ & $7.02 \%$ \\
\hline
\end{tabular}

Maximum absolute values of the VaR and ES measure at $99 \%$ confidence level at five different dates for the three portfolios (EH: empirical histogram).

In summary, the bank supervisors propose to use the Value at Risk as the measure of market risk as a first step, but it appears reasonable to consider other measures in light of the previous analysis. The subprime crises started in 
July $2007^{5}$ have confirmed that the VaR measure is not informative enough to prevent all risks such as the liquidity risk that we are experiencing (see footnote 5). Here we have considered the ES measure but the maximum drawdown as well as the Omega function could be interesting alternatives to $\mathrm{VaR}$. The Omega function, for instance, has been recently investigated in several papers with promising results; see Gilli and al. (2006), Keating and Shadwick (2002) and Caillault and Monier (2009). Now, the banks have to continue with the rules imposed by the Basel amendment, taking into account the variability of the tools proposed. From our point of view all these factors reinforce the uncertainty on the market risk measurement and do not stabilize the financial system as initially planned by the regulators.

\section{References}

Artzner P., F. Delbaen, J. Eber and D. Heath, 1997. Thinking Coherently. Risk, 10, 68-71.

Basel Committee on Banking Supervision, 1996, Amendment to the Basel Capital Accord to incorporate market Risk. BIS, Basel, Switzerland.

Bollerslev T., 1986. Generalized Autoregressive Conditionnal heteroscedasticity. Journal of Econometrics, 31, 307-327.

Brummelhuis R. and D. Guégan, 2005. Multi-period Conditional Distribution for Heteroscedastic Models with Application to VaR. The Journal of Applied Probability, 42, 110-130.

Caillault C. and D. Guégan, 2005. Empirical Estimation of Tail Dependence using Copulas: application to Asian Markets. Quantitative Finance, 5, $489-201$.

Caillault C. and S. Monier, 2009. Copulas and Risk Measures for Strategic Asset Allocation. A Case Study for Central Banks and Sovereign Wealth Funds. Joint BIS/ECB/World Bank Conference "Strategic Asset Allocation for Central Banks and Sovereign Wealth Managers", to be published in summer 2009 by Palgrave MacMillan.

Christoffersen F., F.X. Diebold and T. Schuermann, 1998. Horizon Problems and Extreme Events in Financial Risk Management, Economic Policy Review, 4, 109-118.

Dias A. and P. Embrechts, 2004. Dynamic Copula Models for Multivariate High-Frequency Data in Finance. In: Risk Measures for the 21st

\footnotetext{
${ }^{5}$ At this stage (March 09), the crisis is not over.
} 
Cyril Caillault, Dominique Guégan - Forecasting VaR and Expected Shortfall using Dynamical Systems: A Risk Management Strategy - Frontiers in Finance and Economics - Vol. 6 No.1 - April 2009,

Century, ed. by Giorgio Szegoe, Chapter 16, 321-335. (Wiley Finance Series).

Fermanian J.D, 2005. Goodness of fit tests for copulas. Journal of Multivariate Analysis, 95, 119 - 152.

Francq C. and J.M. Zakoian, 2001. Stationarity of Multivariate MarkovSwitching ARMA models. Journal of Econometrics, 102, 339-364.

Guégan D., 2005. How can we define the concept of long memory? An econometric survey. Econometric review, 24, (2).

Guégan D., 2007a. La persistance dans les marchés financiers, Revue Banques et Marchés, 90, 34 - 43.

Guégan D., 2007b. Global and Local Stationary Modelling in Finance. Submitted to Econometrics Review. www.ces.ens-cachan.fr.

Guégan D. and J. Zhang (2009) Pricing Bivariate Option under GARCH-GH Model with Dynamic Copula: Application for Chinese Market, to appear in European Journal of Finance.

Germat C. and R. D. Harris, 2001. Robust conditional variance estimation and value-at-risk, Journal of Risk, 4, 25 - 41.

Hamilton J., 1988. Rational Expectations Econometric Analysis of Changes in Regime: An Investigation of the Term Structure of Interest Rates. Journal of Economic Dynamics and Control, 12, 385-423.

Huang Y. C. and B.J. Lin, 2004.Value-at-Risk Analysis for Taiwan Stock Index Futures: Fat Tails and Conditional Asymmetries in Return Innovations. Review of Quantitative Finance and Accounting, 22, 79 95.

Gilli, M., E. Këllezi and H. Hilda, 2006. A Data-Driven Optimization Heuristic for Downside Risk Minimization. Journal of Risk, 8 (3), 119.

Keating C. and W. F. Shadwick, 2002. A Universal Performance Measure. The Finance Development Centre.

Joe H., 1997. Multivariate Models and Dependence Concepts. (Chapman \& Hall, London).

Jondeau E. and M. Rockinger, 2006, Conditional Dependency of Financial Series: The Copula-GARCH Model. Journal of International Money and Finance, 25, $827-853$.

Malevergne Y.and D. Sornette, 2003. Testing the Gaussian copula hypothesis for financial assets dependences. Quantitative Finance, 4, 231 - 250.

Mikosch T.and C. Starica, 2004. Non-Stationarities in financial time series, the long range dependence and the IGARCH effect. The review of economics and Statistics, 86, 378 - 390. 
Nelsen R., 1999. An Introduction to Copulas. Lectures Notes in Statistic 139. (Springer Verlag, New York).

Oakes D., 1994, Multivariate Survival Distribution. Journal of Nonparametric Statistics, 3, 343-354.

Patton A. J., C. W. J. Granger and T. Teräsvirta, 2006. Common Factors in Conditional Distributions for Bivariate Time Series. Journal of Econometrics, 127, 43 - 57.

RiskMetrics Technical Document, 1996. (J.P. Morgan, 4th Edition, New York).

Rockafellar R.T. and S. Uryasev, 2002. Conditional Value-at-Risk for General Loss Distributions. Journal of banking and finance, 26, 1443-1471.

Sklar A., 1959, Fonctions de Répartition à $n$ Dimensions et leurs Marges. Publications de l'Institut de Statistique de L'Université de Paris, 8, 229-231.

Yamai Y. and T. Yoshiba (2002), Comparative Analyses of Expected Shortfall and Value-at-Risk (3): Their Validity under Market Stress. Monetary and Economic Studies, Bank of Japan, 20, 181-238.

\section{Appendix: Descriptive Statistics}

Table 5 Descriptive statistics for the three processes $\left(r_{1, t}\right)_{t},\left(r_{2, t}\right)_{t}$ and

\begin{tabular}{|lccc|}
\multicolumn{4}{c}{$\left(r_{3, t}\right)_{t}$} \\
& $\left(r_{1, t}\right)_{t}$ & $\left(r_{2, t}\right)_{t}$ & $\left(r_{3, t}\right)_{t}$ \\
\hline Mean & $-1.34 \times 10^{-4}$ & $-4.33 \times 10^{-5}$ & $-1.00 \times 10^{-4}$ \\
Standard & & & \\
deviation & 0.020 & 0.019 & 0.026 \\
Kurstosis & 5.897 & 26.928 & 40.391 \\
Skweness & 0.014 & 0.023 & 0.838 \\
\hline Jarque-Bera test & 5827 & 121508 & 273992 \\
$D f$ & 2 & 2 & 2 \\
p-value & 0 & 0 & 0 \\
\hline & $\left(\left(r_{1, t}\right)_{t},\left(r_{2, t}\right)_{t}\right)$ & $\left(\left(r_{1, t}\right)_{t},\left(r_{3, t}\right)_{t}\right)$ & $\left(\left(r_{2, t}\right)_{t},\left(r_{3, t}\right)_{t}\right)$ \\
\hline Jarque-Bera test & 127336 & 279820 & 395501 \\
$D f$ & 4 & 4 & 4 \\
p-value & 0 & 0 & 0 \\
\hline
\end{tabular}


Cyril Caillault, Dominique Guégan - Forecasting VaR and Expected Shortfall using Dynamical Systems: A Risk Management Strategy - Frontiers in Finance and Economics - Vol. 6 No.1 - April 2009,

Descriptive statistics for the three processes $\left(r_{1, t}\right)_{t},\left(r_{2, t}\right)_{t}$ and $\left(r_{3, t}\right)_{t}$ (full period $02 / 07 / 87-17 / 12 / 02, \quad N=4033$ observations). The Jarque-Bera test critical value at $5 \%$ is 5.991 for Gaussianity under the null assumption. 